\title{
A Pilot Telerehabilitation Program: DELIVERING EARLY INTERVENTION SERVICES TO RURAL FAMILIES
}

\author{
JANA CASON ${ }^{1}$ \\ ${ }^{1}$ AUERBACH SCHOOL OF OCCUPATIONAL THERAPY, SPALDING UNIVERSITY, LOUISVILLE, KY
}

\section{ABSTRACT}

The enTECH Telerehabilitation Program explored the use of telerehabilitation as an alternative service delivery model for early intervention therapy services. Utilizing the Kentucky Telehealth Network, two families living in rural Kentucky received occupational therapy services over a 12-week period. Following program implementation, qualitative data was collected using participant journals and interviews. Data analysis identified three thematic categories related to the program: benefits/strengths, challenges/weaknesses, and recommendations for program improvement. Results of the program evaluation indicated that telerehabilitation has the potential to cost-effectively meet the therapeutic needs of children living in rural areas where provider shortages exist. The enTECH Telerehabilitation Program serves as a model for how telerehabilitation can be used to deliver early intervention services to ameliorate health disparities and improve access to rehabilitation services.

Keywords: Telerehabilitation, Occupational Therapy, Rural, Early Intervention, Children, Program Evaluation, Cost Analysis

\section{INTRODUCTION}

\section{HEALTH DISPARITIES IN RURAL POPULATIONS}

Sixty-five million Americans, $16-20 \%$ of the U.S. population, reside in rural areas. Only $9 \%$ of primary care physicians practice in rural areas (Bauer, 2003). While people living in rural communities have less access to care, they have greater need for primary and specialty services due to lifestyle factors. Seven million rural Americans live in poverty, 25\% higher than in urban areas. Statistics also show that a higher percentage of elderly residents, people lacking health insurance coverage, and people with chronic diseases reside in rural communities (Bauer, 2003; U.S. Office of Management and Budget, 2006). A higher percentage of individuals with health care needs living in rural areas coupled with fewer practitioners practicing in rural areas results in barriers to access health care services.

Barriers to accessing health care services in rural communities are a paramount concern in Kentucky because " $43.4 \%$ of Kentuckians live in rural counties, compared to $16.9 \%$ for the U.S." (Samuels, Smith, \& Whitler, 2005, p. 8). The health of Kentucky's rural population is compromised due to inadequate public health infrastructure, health provider shortages in rural communities, high-risk behaviors such as smoking and a sedentary lifestyle, and demographic characteristics impacting utilization of health care services (Gross, 2005; Rural Assistance Center, 2007; Samuels, Smith, \& Whitler, 2005). These challenges are also found in rural populations across the United States.

\section{EARLY INTERVENTION NEEDS IN KENTUCKY}

According to the Kentucky Part C (First Steps) Annual Performance Report for State Fiscal Year 2007 (Federal Fiscal Year 2006/FFY 2006), 7,507 children received early intervention services between July 1, 2006 - June 30, 2007. 
Figure 1 - Source - Kentucky Part C (First Steps) Annual Performance Report (FFY 2006); Monitoring

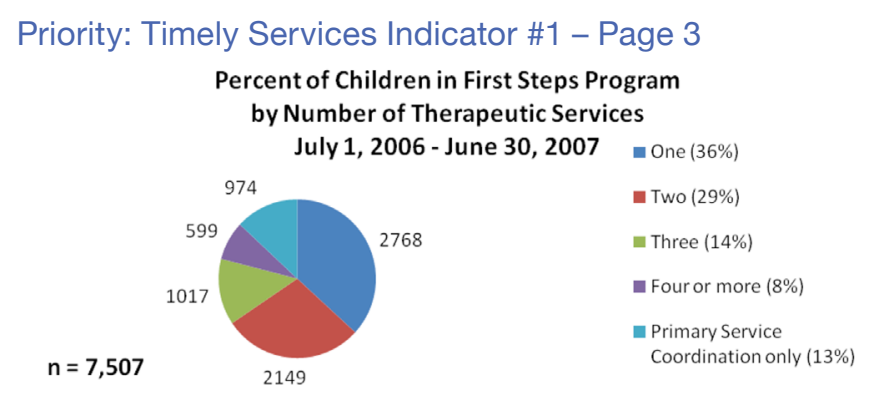

Of these children, 6,533 were identified as needing therapy-based services in addition to primary service coordination. Timely initiation of therapeutic services, within 21 days, occurred for $80 \%$ of the children $(n=5,218)$. The reason for delay in service provision is not well documented but in some cases may be attributed to provider shortages and inadequate access to services.

Figure 2 - Source - Kentucky Part C (First Steps) Annual Performance Report (FFY 2006); Monitoring

Priority: Timely Services Indicator \#1 - Page 2

Percent of Children in First Steps Program with Therapeutic Services within 21 Days July 1, 2006 - June 30, 2007

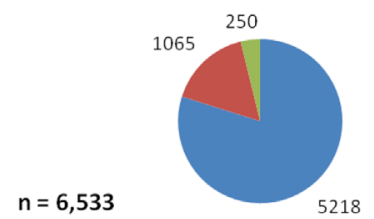

- All services within 21 days $(80 \%)$

- One service more than 21 days $(16 \%)$

- Two or more services more than 21 days $(4 \%)$

\section{TELEHEALTH AND TELEREHABILITATION: A SOLUTION FOR HEALTH DISPARITIES}

Telehealth is an alternative service delivery model that can ameliorate health disparities in rural areas. Telehealth provides real-time interactive encounters with qualified healthcare providers using existing telecommunications technology. Harper (2003) defined telehealth as "the use of advanced telecommunications methods to exchange health information and promote health care across geographic distances and time" ( $p$. 735). Related terms under the "umbrella" of telehealth include telemedicine and telerehabilitation. While these terms are sometimes used interchangeably, there are differences in their meanings. Telemedicine primarily refers to medical service provided by medical practitioners while telerehabilitation refers to services provided by rehabilitation practitioners including occupational therapists, speech therapists, and physical therapists. According to the American Occupational Therapy Association (AOTA), telerehabilitation can be used for intervention planning, implementation, followup care, consultation, education, and supervision of students and other personnel (Wakeford, Wittman, White, \& Schmeler, 2005). Research has verified the efficacy of telehealth, telemedicine, and telerehabilitation as viable options for meeting the medical and rehabilitative needs of individuals living in underserved areas.

Telerehabilitation has been successfully used to provide assistive technology evaluations (Finch, 2000), diagnostic evaluations (Georgeadis, Brennan, Barker, \& Baron, 2004; Harper, 2003), assessment and ongoing therapy services (Clark, Dawson, Schiedeman-Miller, \& Post, 2002; Ziegmann, Cole, Lichtenberg, \& Brooks, n.d.), and community re-entry and support for local practitioners and individuals with disabilities in rural communities (Hagglund \& Clay, 1997; "Telerehab Supports Community Reentry," 2000; Wade, Wolfe, Brown, \& Pestian, 2005). The benefits of telerehabilitation include expert consultation for practitioners and individuals in isolated communities, reduced travel time and related expenses, and increased professional collaboration and rapport (Harper, 2003; Ziegmann, Cole, Lichtenberg, \& Brooks, n.d.).

\section{Kentucky Telehealth Network}

Telehealth began in Kentucky in 1993 with the Kentucky TeleCARE Telehealth initiative developed at the University of Kentucky. In 1997, Medicare legislation enabled reimbursement for designated telehealth encounters. The Kentucky Telehealth Network Board of Directors was established in 2000 through passage of HB 117. The Kentucky Telehealth Network (KTHN), under the leadership of the Board of Directors, currently has over 100 telehealth sites in Kentucky and conducted more than 5,000 clinical telehealth encounters in 2006-07 (Sprang, 2007). Coupled with Kentucky's Department of Public Health technology infrastructure, there are over 200 sites across the state where families could potentially access healthcare services through telehealth encounters.

The Kentucky Telehealth Network provides the infrastructure with its 100 telehealth sites and the ability to link with the Kentucky Department of Public Health videoconferencing network to enable early intervention services to be provided to families within their local communities. Other states, such as lowa, are currently using this model to provide specialized interdisciplinary consultations for children with disabilities. Using the lowa Communications Network (ICN), families travel no more than 15 miles from their homes to link with a specialized interdisciplinary team of professionals at the University of lowa (Harper, 2003). Similarly, utilization of the Kentucky 
Telehealth Network could enable trained early intervention providers to meet the therapeutic needs of children with disabilities within the children's local communities. This alternative service delivery model would be especially beneficial in rural communities where provider shortages limit access to therapy services.

\section{ENTECH TELEREHABILITATION PROGRAM: BACKGROUND AND RATIONALE}

The enTECH Telerehabilitation Program (ETP) grew from this author's professional awareness and personal experience with early intervention provider shortages across the state of Kentucky. As a parent who had experienced the impact of provider shortages, ensuring that all children receive appropriate therapy services from trained providers became a paramount concern. A partnership with Enabling Technologies of Kentuckiana (enTECH), a state-designated assistive technology resource center located on the campus of Spalding University in Louisville, Kentucky led to the development, implementation, and evaluation of a model telerehabilitation program. The research was funded by a grant from the Foundation for a Healthy Kentucky.

\section{Program DeVelopment PHASE}

The development phase of the ETP included three components: (a) literature review and interviews with telerehabilitation researchers; (b) exploration of telerehabilitation technology; and (c) establishment of the ETP's vision, mission, policies, and procedures.

\section{LITERATURE REVIEW AND INTERVIEWS}

Development of the ETP began with a thorough review of literature related to telehealth and telerehabilitation from professional journals and other sources. Over 90 articles formed a foundation for the ETP development. In addition, five interviews were conducted with telehealth program directors and telerehabilitation researchers from across the United States and Europe. Three of interviews were audio recorded and transcribed, providing in-depth information for program development. Internet sources included the American Telemedicine Association, Office for Advancement of Telehealth, and the Telemedicine Information Exchange.

\section{EXPLORATION OF TELEREHABILITATION TECHNOLOGY}

The second component of the development phase involved exploration and piloting of telecommunications technology. To identify the most appropriate technology for the ETP, the researcher piloted five technology options, including the NextLINK 550 Satellite Pro XLS videophone, Skype software with web camera, Sightspeed software with web camera, Windows Live Messenger software with web camera, and utilization of the Kentucky Telehealth Network (KTHN) video conferencing system.

The NextLINK 550 Satellite Pro XLS videophones were piloted during speech therapy sessions with a three year-old child, the researcher, and a licensed speechlanguage pathologist. Strengths of the videophone included minimal technology infrastructure requirements and ease of use. The videophone is small, portable, and has good voice quality. However, the video quality was poor, particularly for facial and body movements. The videophone operates through a standard phone jack, and required disconnecting all other phones from phone jacks within the home environment. The cost (approximately $\$ 2,500$ ) and the challenges encountered during piloting led the researcher to reject the use of videophones for the implementation phase of the ETP.

Videoconferencing opportunities exist through the Internet using a web camera and free software. As part of the development phase, the author explored the use of Skype, Sight Speed, and Windows Live Messenger. Over eight hours of videoconferencing occurred using these three software programs. Two of the interviews with telehealth researchers occurred using Skype. Of the three videoconferencing providers, the researcher found Skype to be the most reliable and effective for telehealth purposes.

A major strength of Internet-based videoconferencing is the ability to provide telehealth services to individuals in their natural environments. Additionally, voice and picture quality were adequate for telehealth purposes. The challenges associated with Internetbased videoconferencing are the need for adequate technology infrastructure (Broadband/High-speed Internet connections) and access to a computer with Windows 98, Windows XP, or Vista. Such challenges can be very frustrating and limiting. For example, a potential participant, a family seeking speech therapy services during the development/web-based implementation phase of the ETP, did not have a computer compatible with the web camera provided for the project and was, therefore, unable to participate in this aspect of the project. According to ConnectKentucky (2007), the state's infrastructure allows $90 \%$ of Kentuckians access to Broadband services. However, the reality is that while infrastructure is in place, access continues to be limited by socio-economic and educational barriers. For this 
reason, the final technology to be piloted and ultimately implemented in the enTECH Telerehabilitation Program was the Kentucky Telehealth Network's videoconferencing system. This existing infrastructure provides state-wide access and circumvents many of the barriers associated with utilization of in-home technology.

\section{ESTABLISHMENT OF ETP'S VISION, MISSION, POLICIES, AND PROCEDURES}

The third component of the development phase of the enTECH Telerehabilitation Program involved establishing the program's vision, mission, policies, and procedures. Integrating information from the literature review and interviews with researchers and telehealth program directors formed a basis for this process. A comprehensive description of the enTECH Telerehabilitation Program's mission, vision, services, referral and scheduling procedures, and implementation strategies, policies, and procedures were established.

\section{PROGRAM IMPLEMENTATION PHASE}

The implementation phase began with the identification of two families in southeastern Kentucky who had children who were eligible for early intervention services but were experiencing barriers to access due to provider shortages. Both families were receiving occupational therapy services from an early intervention provider in Louisville, Kentucky, who traveled 400 miles roundtrip once per month to provide services to the children. Both the therapist and families agreed that the children would benefit from more frequent face-to-face therapy; however, this was not an option. The occupational therapist explained the enTECH Telerehabilitation Program and invited the families to participate. Both families agreed and signed an informed consent agreement. The researcher sent each family a follow-up letter that described their role in the project, and provided information related to the location and contact person for the telerehabilitation encounters. Each family was also provided a journal to record their impressions of the telerehabilitation experience. The journals were collected at the conclusion of the project's implementation phase and were used to supplement information from participant interviews.

Consultative occupational therapy services commenced in November 2007 and continued over a 12-week period. Eight therapeutic encounters were planned during the implementation phase; however, due to weather and illness, a total of six therapeutic encounters occurred. The children's occupational therapist connected with the families within their local community from a Kentucky Telehealth Network (KTHN) site in Louisville,
Kentucky. The participating families connected with the occupational therapist at a partnering KTHN site within their local community. Onsite technical support at each location initiated the videoconference connection and assured that the cameras were correctly positioned. During the therapy encounter, however, only the therapist, caregiver, and child were in the room at their respective locations. The occupational therapist provided consultative therapeutic services directly to the caregiver and child and facilitated the caregiver's interactions with the child remotely. Each therapeutic encounter lasted approximately 30 minutes.

\section{PROGRAM EVALUATION PHASE}

The purpose of the enTECH Telerehabilitation Program (ETP) evaluation was to determine if early intervention occupational therapy services could be delivered using advanced telecommunications technology. Purposive sampling was used to elicit opinions from participants in the ETP. Qualitative data, including interviews with participants and analysis of participants' journals, were collected and analyzed to determine strengths and weaknesses of the ETP and to make recommendations for changes and future applications of the program. Interviews were originally to be conducted using the KTHN; however, due to illness and scheduling conflicts, the interviews occurred over the telephone with the researcher, implementing occupational therapist, and individual ETP participants. Prepared, open-ended questions elicited information from participants about personal challenges related to accessing needed therapy services; expectations and level of satisfaction with telerehabilitation encounters; strengths and weaknesses of the ETP; and recommendations for program changes. Each participant interview was audio recorded and transcribed. Both the interview transcripts and journal entries were analyzed to identify preliminary categories and common themes related to the ETP experience. To assure trustworthiness of results, member checking and triangulation of data (cross referencing interview transcripts with participant journals) were implemented (Gilchrist \& Williams, 1999).

The data analysis process for the enTECH Program Evaluation incorporated four different analysis cycles. The first analysis cycle occurred as data was collected through the interview process and the researcher had an opportunity to reflect immediately following as well as between participant interviews. The second analysis cycle occurred as the interview transcripts were individually analyzed and coded. Interpretive notations and observations were made in the margins of the transcript to initially categorize chunks of data. Initial categories were: a) access; b) therapy; c) ETP experience; and d) program-related recommendations. These chunks of data were re-coded based on similar concepts within 
the categories. Frequency of code occurrences provided a basis for identifying key areas for further analysis. The identified themes were: challenges with access; benefits of therapy; challenges/weaknesses of ETP; benefits/ strengths of ETP; recommendations for improvement; and considerations for future application. The third analysis cycle involved analyzing the ETP participant journals. Participants' journals were read thoroughly and individually coded. The coded data was organized into the existing themes that had emerged from the interview transcript analysis process. No additional themes emerged during this process. The fourth analysis cycle involved linking similar concepts/themes to understand the experience of parents who had experienced barriers to accessing early intervention services, participants' perceptions of the ETP (including the perception of the occupational therapist involved with implementation), and recommendations for program improvement.

This analysis cycle resulted in the original six themes collapsing into three overarching themes: a) Benefits/ Strengths of ETP, b) Challenges/Weaknesses of ETP, and c) Recommendations for improving ETP.

\section{RESULTS}

\section{ETP BENEFITS AND STRENGTHS}

The ETP program enabled the two pilot families to supplement their monthly in-person occupational therapy encounters with telerehabilitation encounters. One parent identified the opportunity to receive additional therapy services as an incentive for participating in the program. She stated, "Instead of just once a month, we were able to see each other two or three times and it did help... (my child)." Telehealth encounters enabled the ETP-based occupational therapist to provide timely recommendations for families as the children mastered new skills. This is significant because without this technology, the families would have waited a month before learning new intervention strategies.

The results of the enTECH Telerehabilitation Program evaluation are encouraging. Both parent participants reported a high level of satisfaction with telerehabilitation and expressed the belief that their children benefited from participation. A larger research project that incorporates multiple therapy disciplines and participants from geographical regions across the state is now recommended. The comparison of quantitative data from state approved assessments administered prior to and immediately following participation could provide useful information on the efficacy of telerehabilitation as an alternative service delivery model for early intervention services.

\section{ETP CHALLENGES AND SOLUTIONS}

The primary challenge identified by the participants, including the implementing therapist, related to the environment and room arrangement for the telerehabilitation encounters. Ideally, the room setup for telerehabilitation encounters would allow ample space for the child and caregiver to play in multiple positions within view of the camera. Access to an area on the floor with toys, a high chair, and other positioning devices used in the home such as a standing frame or therapeutic wedge would provide a greater variety of therapeutic opportunities during the telerehabilitation encounter. Additionally, having the same equipment and toys available at both initiating and community sites would allow the therapist to demonstrate play activities, set-up of positioning devices, and proper positioning of the child within the devices, perhaps using a doll for simulation. Efforts were made to improve the set-up of the rooms where the telerehabilitation encounters occurred, however, this continued to be a limitation of the ETP due to the short duration of the project and limited ability to reconfigure the room at the community site.

Both families expressed interest in technology that would enable their children to receive telerehabilitation services within the home environment. Transportation challenges, compromised immune system and prevention of illness, increased comfort level of children in their homes, and availability of objects needed during therapy encounters were sighted as potential benefits of this option. Optimal encounters would enable families to access toys, assistive technology, and other items needed for children to practice specific skills related to Individualized Family Service Plan (IFSP) outcomes under the direction and guidance of the therapist.

\section{COST-SAVINGS ANALYSIS}

Utilization of telerehabilitation for early intervention services for children where provider shortages exist would result in a cost savings for Kentucky. Savings are realized even when increased utilization of early intervention services is calculated into the formula. First, it is important to recognize that the United States federal government outlines a specific percentage of infants and toddlers birth to three who should have Individualized Family Service Plans (IFSPs) based on comparisons with other states with similar eligibility definitions and national data. Nationally, $2.43 \%$ of children from birth to three have IFSPs. According to the Kentucky Part C (KEIS/First Steps) Annual Performance Report for State Fiscal Year 2007 (Federal Fiscal Year 2006/FFY 2006), the measurable and rigorous target for the percentage of children with IFSPs from birth to three in Kentucky was $2.40 \%$. As indicated in Figure 3, the actual percentage of 
children with IFSPs from birth to three in Kentucky for FFY 2006 was $2.26 \%$ ( $n=3786)$. These numbers indicate that Kentucky is currently falling short of its target. Identifying and implementing services for a larger percentage of children birth to three, an additional $0.14 \%$, would allow Kentucky to reach this target. However, access to services is limited for many children across the state due to provider shortages. This shortage would likely increase if additional children as outlined in the target outcome were to enter the First Steps program.

Figure 3 - Percent of Population Birth to Three with IFSPs

\section{Percent of Population Birth to Three with IFSPs}

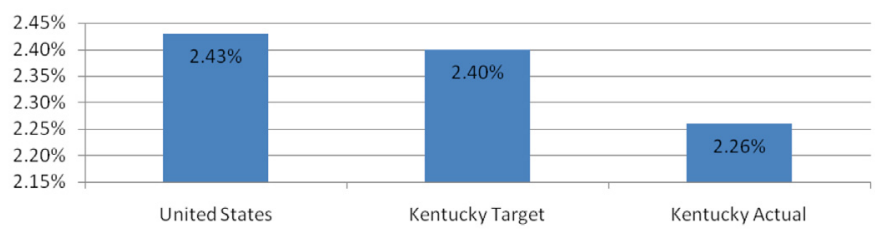

(Data Source - Kentucky Part C Annual Report. (2008). Part C State Annual Performance Report (APR) for FFY 2006, Monitoring Priority: Child Find Indicator \#6 - Page 1-2)

By using telerehabilitation, provider shortages could be alleviated and more children could receive therapy services without creating an increased financial burden for the First Steps program. An actual case example outlines this reality. The ETP implementing occupational therapist currently travels to southeastern Kentucky, a 400-mile round trip, one time per month to provide occupational therapy services to children in the KEIS program who would otherwise not receive services due to provider shortages in that area. Travel costs incurred total $\$ 462.00$ and are calculated as follows: Mileage = $\$ 194.00$ (400 miles $\times \$ 0.485$ per mile); Hotel $=\$ 178.00$ $(\$ 89.00 \times 2$ nights); Meals $=\$ 90.00$ ( $\$ 45.00$ per day). The occupational therapist provides an average of 15 hours of therapy per trip reimbursed at a rate of $\$ 89.00$ /hour totaling $\$ 1335$ paid by Kentucky for services rendered. These numbers reflect the true costs for providers to service rural areas and reveal that travel expenses account for $35 \%$ of the reimbursed rate for therapy services in this example (Figure 4). While Kentucky does not incur the travel costs, there has been discussion to provide an incentive, at an increased cost to the state, for providers to serve rural areas where provider shortage exists. At the very least, this example provides a glimpse of the costs incurred by providers when traveling to rural areas and highlights the reason that many rural areas are experiencing provider shortages.
Figure 4 - Cost incurred for travel from Louisville to Hazard, Kentucky; 15 billed therapy hours

Costs incurred for travel from Louisville to Hazard, KY for therapy encounters

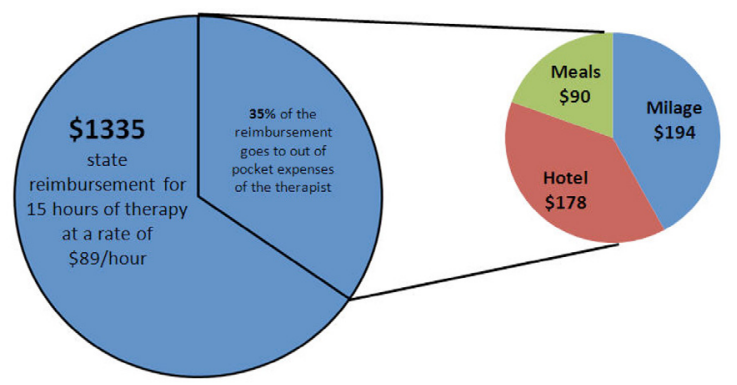

Therapists traveling to rural areas to provide therapy services sacrifice time in addition to incurring travel costs. In the above example, the occupational therapist spends a minimum of nine hours of travel time, including roundtrip from Louisville to southeastern Kentucky and travel between children's homes. While it is difficult to quantify this non-reimbursed cost, it is important to note that these nine hours could better be spent providing actual therapy encounters for children needing services.

A comparison of community-based therapy services (reimbursed by Kentucky at a rate of $\$ 89.00 /$ hour) versus telerehabilitation services (reimbursed by the Kentucky at the 'office based' service rate of $\$ 63.00 /$ hour) reveals that telerehabilitation could result in a cost-savings. For example, the occupational therapist in this example is currently reimbursed $\$ 1335.00$ by Kentucky for 15 hours of community-based occupational therapy services. If the therapist provided these same services using telerehabilitation, their reimbursement rate would be $\$ 945.00$, a savings of $\$ 390.00$. Additional savings may occur through telerehabilitation due to sessions being completely consultative-based and more focused leading to shorter (30-45 minutes) sessions versus traditional one-hour sessions. If the occupational therapist provided 45-minute sessions via telerehabilitation with these 15 children, their reimbursement rate would be $\$ 708.00$, a savings of $\$ 626.25$ when compared to one-hour community-based encounters (Figure 5).

Figure 5 - Cost comparison of community vs. telehealth services

Cost comparison of community vs. telehealth services

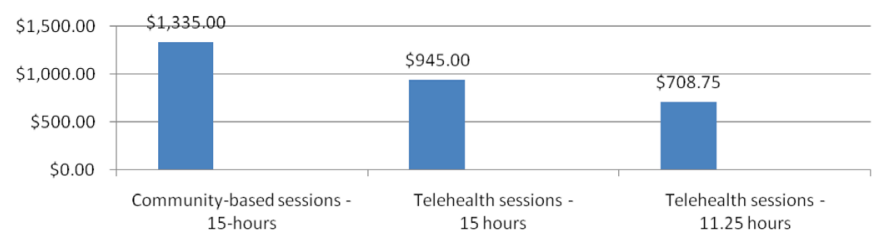


Telehealth is clearly cost-effective and improves access for children in rural areas where provider shortages exist. Statistics indicate that $87 \%$ more children could receive therapy services without increasing cost. Using telerehabilitation, six additional children ( $n=21)$ could receive one-hour of occupational therapy services. If the telerehabilitation encounters were fortyfive minutes, rather than one-hour, 13 additional children $(n=28)$ could receive occupational therapy services using telerehabilitation at no additional cost for the KEIS program (Figure 6).

Figure 6 - Number of children serviced for approximately the same cost (\$1335.00)

Number of children serviced for approximately the same cost $(\$ 1335.00)$

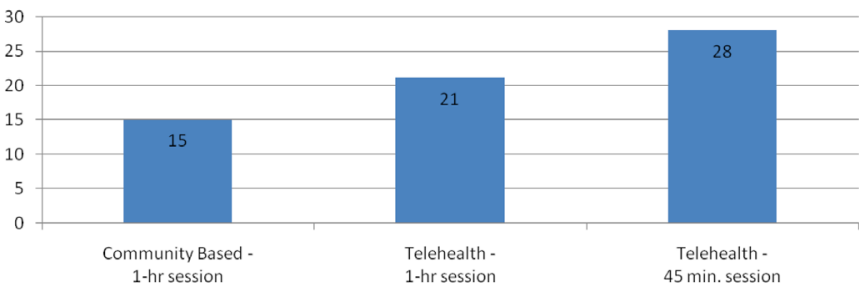

Clearly, utilizing telerehabilitation to provide therapy services to children living in areas where provider shortages exist could result in a cost-savings for Kentucky's early intervention program. Provider shortages impact both quantity and quality of services children receive. As a result of provider shortages, therapists in the field report that children either receive no service, services by another provider outside the discipline of need, or services by a local provider without pediatric experience/training. By providing targeted interventions using skilled pediatric practitioners, children should reach desired outcomes faster with fewer therapy sessions. Telerehabilitation has the ability to provide a service to children in rural areas who would otherwise not have access; to support therapists from other disciplines in implementing strategies related to the discipline of need (trans-disciplinary services); and to provide expertise and support to local providers who lack pediatric training and/ or experience.

\section{FUTURE DIRECTIONS}

Telerehabilitation has the potential to alleviate provider shortages and improve access to early intervention services. Utilization of the Kentucky Telehealth Network and the Department of Public Health videoconferencing capabilities would enable families in provider-shortage areas to tap into the expertise of pediatric therapists from across the state without leaving their local communities. Telerehabilitation could also support local practitioners who desire collaboration with therapists who have specialized knowledge in a specific area such as feeding or sensory integration. Kentucky's Early Intervention System has adopted a consultative service delivery model and supports collaboration among providers. Telerehabilitation employs this same delivery model to enable distant therapists to serve as 'brokers of knowledge' for families and local providers.

Exploring technology such as videophones and webbased technology for service delivery within the home would be beneficial. However, due to socio-economic and educational barriers, the Kentucky Telehealth Network and the Department of Public Health technology may prove optimal for service delivery on a state-wide level. The infrastructure and technology currently available provides state-wide access and would require minimal capital investment or training.

If telerehabilitation is adopted by Kentucky's Early Intervention System as an alternative service delivery model, it is critical that providers using this technology have adequate training and pediatric experience. The researcher recommends that telerehabilitation be limited to providers with a minimum of two years pediatric experience, preferably in the early intervention setting. This requirement would help ensure that providers have the necessary skills and experience to overcome the challenges associated with directing caregivers and demonstrating techniques without being physically present. Mandatory training that provides information and skill development related to telerehabilitation technology and therapeutic considerations is also recommended. Ideally, only providers who meet the pediatric experience and mandatory training requirements would be able to utilize and be reimbursed for early intervention telehealth encounters. As providers gain experience using telerehabilitation, a mentoring program could be established to develop the skills and abilities of prospective telehealth providers. 


\section{SUMMARY AND CONCLUSIONS}

The enTECH Telerehabilitation Program (ETP) resulted in the development, implementation, and evaluation of a pilot telerehabilitation program that provided early intervention occupational therapy services to children living in rural Kentucky. The development phase included a thorough literature review, interviews with telerehabilitation researchers and program directors from across the United States and Europe, exploration of telecommunication technology, and development of policies and procedures for program implementation. Utilizing the Kentucky Telehealth Network, two families living in rural Kentucky received early intervention occupational therapy services over a 12-week period during the implementation phase of the ETP. Following implementation, qualitative data was collected from participant journals and interviews. The interviews were transcribed and coded to identify overarching themes, and participant journals were used to triangulate data from the interviews. Results of the program evaluation were positive and indicate that telerehabilitation does have the potential to meet the therapeutic needs of children living in rural areas. A cost-savings analysis further supports the use of telerehabilitation to meet the therapeutic needs of children living in rural areas.

Exploration of telerehabilitation options for early intervention services where inadequate access to providers exists is critical. Provider shortages impact the quantity and quality of services available in Kentucky for children with special needs, particularly in rural areas. The results of the enTECH Telerehabilitation program evaluation demonstrated the potential of telerehabilitation to support families, children, and providers within their local communities. A cost-savings analysis indicates that telerehabilitation services could enable therapists to serve more children without increasing costs. The enTECH Telerehabilitation program serves as a model for how telerehabilitation could be delivered through telecommunications technology by trained early intervention providers to ameliorate health disparities and improve access to early intervention services.

\section{ACKNOWLEDGEMENTS}

This project was supported by The Foundation for a Healthy Kentucky, Louisville, KY. (http://www.healthyky. org) The views expressed in this report are those of the author and may not reflect the views of the Foundation, its governing board, or staff. (http://www.healthyky. org) The author would like to thank the Foundation for a Healthy Kentucky for supporting this project; the many researchers who willingly agreed to be interviewed and shared their time, knowledge, and expertise as part of the development phase of the enTECH Telerehabilitation program; Rob Sprang, Tim Bickel, and Beth Howard who shared their expertise and opened their facilities for the implementation phase; Debbie Thompson, OTR/L for her work in the implementation phase of the program; the families who participated; Dr. Susan Meyers for her review and support of this project; Dr. Laura Strickland for her leadership and support; Dr. Randy Strickland for his mentorship and guidance throughout this project; and Terri Schoone and Judy Luther for their editorial assistance. 


\section{REFERENCES}

Bauer, K. (2003). Distributive justice and rural healthcare: A case for e-health.

International Journal of Applied Philosophy, 17(2), 241-252.

Clark, P., Dawson, S., Scheideman-Miller, C. \& Post, M. (2002). Telerehab: Stroke teletherapy

and management using two-way interactive video. Neurology, 26(2), 87-93.and

ConnectKentucky. (2007). Connect Kentucky 2007 progress report. Retrieved March 24, 2008,

from http://www.connectkentucky.org/_documents/ connectkentucky_2007.pdf

Finch, L. (2000). Ingenious engineering. Rehab Management, 104-110.

Georgeadis, A., Brennan, D., Barker, L., \& Baron, C. (2004). Telerehabilitation and its

effects on story telling by adults with neurogenic communication disorders.

Aphasiology, 18, 639-652.

Gilchrist, V. \& Williams, R. (1999). Key informant interviews. In B. Crabtree \& W. Miller (Eds.),

Doing qualitative research (2nd ed.) (pp. 71-88). Thousand Oaks, CA: Sage.

Gross, D. (2005, Summer). KRHA conference: State of KY's health 'bad', panelists say.

Rural Health Update, 2.

Hagglund, K. \& Clay, D. (1997). Rural healthcare initiatives in spinal cord injury.

American Rehabilitation, 23(1), 2-6.

Harper, D. (2003). Telehealth. In. M. Roberts (Ed.), Handbook of Pediatric Psychology

(3rd ed., pp. 735-746). New York: Guilford Press

Kentucky Part C Annual Report. (2008). Part C State

Annual Performance Report (APR) for FFY

2006. Retrieved March 20, 2008, from http://chfs. ky.gov/NR/rdonlyres/A1D33FFF-C84C-432B-86FB1468461C2F92/148765/FFY06APRRevised.pdf

Rural Assistance Center. (2007). Kentucky. Retrieved April 5, 2007, from

http://www.raconline.org/states/kentucky.php

Samuels, M., Smith, J., \& Whitler, E. (2005, Summer).

Focus on: Rurality - Place of

residence shown to have impact on health status. Rural Health Update, 8-9.

Sprang, R. (2007, December). Kentucky Telehealth

Network (KTHN):2006-2007 annual report.

Paper presented at the meeting of the Interim Joint Committee on Health and Welfare Kentucky

Telehealth Network Board of Directors, Frankfort, KY.

Telerehab supports community reentry. (2000, October). Case Management Advisor,

164-166.

U.S. Office of Management and Budget. (2006). Detailed information on the Rural Health

Activities Assessment. Retrieved on April 5, 2007, from

http://www.whitehouse.gov/omb/expectmore/ detail.10001065.2005.html

Wade, S., Wolfe, C., Brown, T., \& Pestian, J. (2005).

Putting the pieces together:
Preliminary efficacy of a web-based family intervention for children with traumatic

brain injury. Journal of Pediatric Psychology, 30(5), 437442.

Wakeford, L., Wittman, P., White, M., \& Schmeler, M. (2005). American Occupational

Therapy Association (AOTA) Telerehabilitation position paper. Retrieved February 25,

2009, from http://www.aota.org/Practitioners/Official/ Position/36203.aspx

Ziegmann, M., Cole, E., Lichtenberg, A., \& Brooks, R. (2001). Telerehabilitation: New tools for

providing in-home brain injury rehabilitation. Retrieved February 25, 2009,

from http://www.aota.org/Pubs/OTP/1997-2007/ Features/2001/f-110501.aspx 
